Proceedings of the Third International Conference on

\title{
Wavelet Analysis and Its Applications (WAA]
}

\author{
Volume 1
}




\title{
Supported by
}

\author{
National Nature Science Foundation of China (NSFC) \\ Chinese Mathematics Association \\ Foreign Affairs Bureau of General Logistical Ministry of PLA \\ Chongqing People Affairs Bureau \\ Chongqing Electronic Association
}


Proceedings of the Third International Conference on

\title{
Wavelet Analysis and Its Applications (WAA]
}

Chongqing, PR China $\quad 29-31$ May 2003

\section{Volume 1}

\author{
Editors \\ Jian Ping Li \\ Logistical Engineering University, PR China \\ Victor Wickerhauser \\ Washington University, USA \\ Yuan Yan Tang \\ Hong Kong Baptist University, Hong Kong \\ John Daugman \\ University of Cambridge, UK \\ Lizhong Peng \\ Peking University, PR China \\ Jing Zhao \\ Logistical Engineering University, PR China
}




\section{Published by}

World Scientific Publishing Co. Pte. Ltd.

5 Toh Tuck Link, Singapore 596224

USA office: Suite 202, 1060 Main Street, River Edge, NJ 07661

UK office: 57 Shelton Street, Covent Garden, London WC2H 9HE

\section{British Library Cataloguing-in-Publication Data}

A catalogue record for this book is available from the British Library.

\section{WAVELET ANALYSIS AND ITS APPLICATIONS (In 2 Volumes) Proceedings of the Third International Conference}

Copyright $\odot 2003$ by World Scientific Publishing Co. Pte. Ltd.

All rights reserved. This book, or parts thereof, may not be reproduced in any form or by any means, electronic or mechanical, including photocopying, recording or any information storage and retrieval system now known or to be invented, without written permission from the Publisher.

For photocopying of material in this volume, please pay a copying fee through the Copyright Clearance Center, Inc., 222 Rosewood Drive, Danvers, MA 01923, USA. In this case permission to photocopy is not required from the publisher.

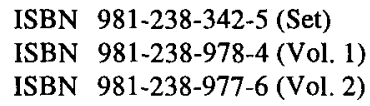

This book is printed on acid-free paper.

Printed in Singapore by Mainland Press 


\section{Preface}

Wavelet analysis and its applications have become one of the fastest growing research areas in the past several years. Wavelet theory has been employed in many fields and applications, such as signal and image processing, communication systems, biomedical imaging, radar, air acoustics, and endless other areas. For the past several years, reflecting the excitement and creativity surrounding the subject of wavelets, articles about wavelets have appeared in professional publications, on World Wide Web, and in professional magazines and newspapers. Much of this enthusiasm for wavelets comes from known and from potential applications. For instance, wavelets have found use in image processing, in the restoration of recordings, and in seismology.

In order to stimulate the future development, explore novel applications, and exchange ideas for developing robust solutions, the Third International Conference on Wavelet Analysis and Its Applications (ICWAA2003) was held at Logistical Engineering University in Chongqing in May 2003. This conference is following the success of the first conference (ICWAA1999) and second conference (ICWAA2001). We have received 195 full papers submitted from all over the world. To ensure the quality of the conference and proceedings, each paper was reviewed by different reviewers. After a thorough review process, the program committee selected 156 papers as regular papers and short papers. The proceedings of ICWAA2003 was divided into volume I and volume II published by World Scientific. There were 3 invited talks delivered by distinguished researchers, namely Prof. John Daugman from Cambridge University, UK, Prof. Bruno Torresani from Inria, France, and Prof. Victor Wickerhauser from Washington University, USA. We must add that the conference organizing committee, the conference program committee and the reviewers did an excellent job within a very tight schedule.

We wish to thank all the authors for submitting their work to ICWAA2003 and all the participants, whether you came as a presenter or an attendee. We hope that there was ample time for discussion and opportunity to make new acquaintances. Finally, we hope that you experienced an interesting and exciting conference and enjoyed your stay in Chongqing.

We hope that you will enjoy and benefit from the papers in this book.

Jian Ping Li, Professor, Ph.D.

Head of International Centre for Wavelet Analysis and Its Applications Logistical Engineering University, Chongqing 400016, P.R.China 
This page is intentionally left blank 


\section{Conference Organization}

\section{Congress General Chair:}

Congguang Lin, Logistical Engineering University

\section{General Chairs:}

John Daugman, Cambridge University

Yuan Yan Tang, Hong Kong Baptist University

Lizhong Peng, Peking University, China

\section{Program Chairs:}

Jian Ping Li, Logistical Engineering University

Victor Wickerhauser,Washington University

Bruno Torresani, INRIA and University de Provence, France

\section{Organizing Committee Chairs:}

Chizhong Bao, Logistical Engineering University

Chengdong Fang, Logistical Engineering University

\section{Publications Chair:}

Jing Zhao, Logistical Engineering University

\section{Organizing Committee:}

Shunxing Fang, Shihai Chen, Xuezhen Li, Qiyue Zhang,

Gang Zhao, Qiang Bao, Youguang Wang, Min Zhang, Fei Chen, Yongjun Zhang, Yueping Zhu, Xianlu Wang, Hongbo Gu, Congbin Yu, Zhanguo Yuan, Shangan Yan, Jing Zhao, Qiong Lin, Xiuwen Yang, Jiangtao Zhai, Wei Pan

\section{Sponsors}

National Nature Science Foundation of China (NSFC)

Chinese Mathematics Association

Foreign Affairs Bureau of General Logistical Ministry of PLA

Chongqing People Affairs Bureau

Chongqing Electronic Association 


\section{Program Committee:}

Metin Akay, Dartmouth College

Akram Aldroubi, Vanderbilt University

Claudia Angelini , Istituto per Applicazioni della Matematica

Fengshan Bai, Jiamusi University

Algirdas Bastys, Vilnius University

T. D. Bui, Concordia University

Elvir Causevic, Everest Biomedical Instrument Company

Mariantonia Cotronei, Universita' di Messina

Hans L. Cycon, Fachhochschule fur Technik und Wirtschaft Berlin

Zhengxing Cheng, Xi'an Jiaotong University

Yunan Chui, Harbin Science and Technology University

Siyuan Cao, Beijing Oil University

Zhongxing Deng, Haerbin Science and Technology University

Dao-Qing Dai, Zhongshan University

Wolfgang Dahmen, Technische Hochschule Aachen

Donggao Deng, Zhongshan University

T. N. T. Goodman, University of Dundee

D. Hardin, Vanderbilt University

Wen-Liang Hwang, Institute of Information Science, Taiwan

Rong-Qing Jia , University of Alberta, Canada

P. Jorgensen, University of Iowa

K. S. Lau, Hongkong Chinese University

Seng-Luan Lee, National University of Singapore, Singapore

Wei Lin, Zhongshan University

Jinzao Lin, Chongqing Information Technology Bureau

Jiaqi Liu, Harbin Science and Technology University

Guixing Luan, Shenyang Inst. of Computing Technology

Hong Ma, Sichuan University

Peter Oswald, Bell Laboratories, Lucent Technologies

Valie Perrier, Domaine Universitaire

S. D. Riemenschneider, West Virgina University

Zuowei Shen, National University of Singapore, Singapore

Guoxiang Song, Xi'an Electronical University of Science and Technology

Georges Stamon, University Rene Descartes

Chew-Lim Tan, National University of Singapore, Singapore

Fengchun Tian, Chongqing University

Michael Unser, Batiment de Microtechnique

Jianzhong Wang, Sam Houston State University, U.S.A 
Yueshen Xu, University North Dakota, U.S.A

Lihua Yang, Zhongshan University

Shouzhi Yang, Xi'an Jiaotong University

Li Zeng, Chongqing University

Rongmao Zhang, Shenyang Inst. of Computing Technology

Jing Zhao, Logistical Engineering University

Qingshen Zhu, Chongqing University

Xingwei Zhou, Nankai University

\section{Conference Secretariat:}

Jing Zhao, Xiuwen Yang, Qiong Lin, Wei Pan 
This page is intentionally left blank 


\section{Contents \\ (Volume 1)}

\section{Keynote Presentations}

Accelerating Convergence of Monte Carlo Simulations and Measuring Weak Biosignals Using Wavelet Threshold Denoising

M. V. Wickerhauser

\section{Image Compression and Coding}

A Novel Solution to Tile Effect Exhibited by Fractal Image

Compression Techniques

C. He, X. Huang, H. Jiang and T. Liu

Global Distortion Optimal Bit Allocation Scheme for Image

Compression

J. M. Wang, M. Z. Mao and Y. Y. Xue

One of Image Compression Methods Based on Biorthogonal Wavelet Transform and LBG Algorithm

J. Lin, G. Jing and X. Guo

Gene, Wavelet, Fractal and Data Compression

F. Tian, Y. Huang, X. Zeng and L. Hong

Design of Biorthogonal Wavelets Based on Lifting Scheme and Its Application to Image Coding

$X$. Ding, $R$. Zhu and J. $\mathrm{Li}$

Two-Dimensional Biorthogonal Wavelet Transform and Its Application on Fingerprint Images Compression

$J$. Lu, X. Wu and Y. Liu

Constructing for Irregular Region in Fractal Image Compression

H. Fan and C. Fan

The Relationship between Wavelet Bases and Image Compression

$X . Y u$ 


\section{Video Coding and Processing}

The Speech Analysis and Segmentation by Discrete Wavelet

Transform (DWT)

J. F. Ma and H. Q. Wang

Application of Bi-Orthogonal Wavelet Transform in the De-Noising of Gyro Signal

$X$. Wang, J. Teng, G. Zhou and S. Li

Wavelet Transform Application of Video Coding at Very Low Bit Rate

$X$. Fu, Z. Wang and D. Liang

A Video Watermarking Algorithm Using Fast Wavelet

J. Zhang, Y. Huang, H.Wang and Z. Zhang

DC Coefficients Recovery-Based Error Concealment Technique for Mpeg-2 Video Streams

J. Cao, Z. Wang and F. Li

Wavelet Denoising of Derivative Near Infrared Spectra (NIR)

G. Tian, H. Yuan, W. Lu and H. Lin

\section{Theory}

Fast Method to Compute Tensor Product 2-D Wavelet Transforms

Y. Sun and L. Tang

Bivariate Nonseparable Scaling Function

$S$. Yang, Z. Cheng and X. Feng

Coherent Structure and its Influence to Scaling Law in Rayleigh-

Bénard Convection Based on Wavelet Transformation

Q. Fu, L. Li and K. Xia

Multi-Frequency Biorthogonal Wavelets Generated by a Finite

Number of Functions

Z. Shi and G. Song

A Novel Adaptive Wavelet via Lifting Scheme

W. Huang, D. Bi, B. Mao and Z. Chen

The Wavelet Approximation in Reproducing Kernel Spaces $H^{1}(I)$

C. Deng

Adapted Sampling and Interpolatory Wavelet Packets 
A Class of Semi-Orthogonal Wavelet Packets

$Y$. Y. Tang, J. Yang and W. Zhang

Wavelet Transform for Multiple Decomposition of Gravity Anomalies Z. Hou and P. Sun

A Fuzzy-Logic-Based Denoising Method in Wavelet Domain

S. Li and L. Liu

Nonorthogonal B-Spline Multiresolution Analysis

N. A. Strelkov

Data Compression by Orthogonal Multiwavelets of Multiplicity Four

L. T. Guan, X. Liu and L. Guan

The Research of Complex System Modeling Based on Fractal Conception

L. Wang, Z. Zuo, Y. Xiao and $Q$. Wu

Generalized Multiresolution Analysis

Y. Guan and Y. Zhou

Structural and Geometric Characteristics of Sets of Convergence and Divergence of Multiple Fourier Series of Functions which Equal Zero on Some Set

I. L. Bloshanskii

Strong Convergence for Generalized Abstract Evolutionary Algorithm

M. Z. Xue, W. C. Zhong and L. C. Jiao

Multiscale Wavelet Texture Based Iris Verification

$L$. Yu, K. Wang, C. Wang and D. Zhang

The Wavelets Characterization of Weighted Triebel-Lizorkin Spaces M. Xu

\section{Image Processing}

Sequence Images Data Fusion Based on Wavelet Transform Approach H. Tao, W. Chen, J. Tian and J. Liu

Skeleton Extraction of Ribbon-Like Shapes Based on Wavelet

Transform 
A Method for Feature Extraction of Target Signal Based on Wavelet Decomposition

L. P. Jiang, S. G. Gong, W. W. Hu and S. B. Wang

A Wavelet Based Watermarking Scheme for Information Security

$J$. Yang, J. Cheng and B. Li

Multiwavelet Thresholding with Context Modeling for Image

Denoising

$X$. Shang, B. Zhang and G. Song

Characters Automatic Detection in Color Image by Wavelet Transform

$Y$. Chen, L. Wang and Z. Wan

Steganalysis of Images Created in Wavelet Domain Using

Quantization Modulation

S. Liu, H. Yao, X. Fan and W. Gao

Iris Image Analysis and Reconstruction Based on Zero-Crossing Wavelet Transform

Y. Liu, Z. Li, S. Yuan and T. Xu

CT Image of Liver Cancer Enhancement Using Wavelet Transform

D. Feng, Z. Wang, Y. Cheng, W. Bao, S. Zhou and Y. Luo

Wavelet-Domain Least Squares Based Image Superresolution

S. Zhao, P. Zhang and S. Peng

Image Fusion Using Wavelet Packet Transform

W. Liu and L. Zhou

A Composite Edge Detector Based on Multi-Wavelet

C. Cai, M. Ding and C. Zhou

Study of Two New Wavelet Templates for Medical Cephalometric Image Processing

Y. Du and D. Wang

Similar Image Retrieval Based on Wavelet Transformation

C. C. Chang, J. C. Chuang and Y. S. Hu

\section{Signal Processing}

Invisible Pattern Recognition and Wavelet Analysis

F. S. Bai, L. Gu and Y. Y. Tang 
Extracting and Analyzing Sub-Signals in Heart Period Signal

Z. Y. Lee, J. H. Yu, T. Y. Xiang, H. X. Ma, Y. H. Niu, Z. X. Xie and J. C. Yang

The Research of Mechanical Fault Diagnosis on Wavelet Packet Transform

H. Wang, J. Li and W. Pan

Robust-M Detection of Signal Based on Wavelet Packet Transform

Q. Xiang, C. Lin and S. Gong

Power Quality Short-Term Disturbance Recognition Using Wavelet

$X$. Xiao and H. G. Yang

Date Fusion for Medical Cephalometric Image Based on Lifting Scheme

D. Wang and $Y$. Du

Recognition of Oceanic Explosion Signals Based on Wavelet Packet

Transform and Neural Network

R. Li, Y. Ma and Z. Zhang

Radar Detection of Minimum Altitude Flying Targets Based on

Wavelet Transforms

H. Li, C. Shang, Y. Huang and Z. Wang

\section{Systems and Application}

GO Methodology for Engineering Risk Analysis

Q. Yong, S. Li, F. Chen, K. Zhu, F. Xiao and J. Fu

Wavelet Transformation on Hidden Markov Models

$M$. Wang and $H$. Ma

An Application of Fault Diagnosis for Bearings in the Gearbox of the

Propelled Gun Using Wavelet

W. Wang, H. Feng and L. Tang

Fourier Analysis on the Measuring of Non-Linear Distortion

S. Yan, J. Zhai and T. Yang

Symmetry Phase Congruency: Feature Detector Consistent with Human Visual System Characteristics

Z. Xiao, M. Yu, Q. Li and C. Guo

The Application of Lifting Scheme in Pitch Detection 
Some Results on Bivariate Nonseparable Wavelets

$X$. Gao and H. Zhong

Wavelet and Support Vector Machines for Short-Term Electrical

Load Forecasting

Y. Li and T. Fang

Wideband Waveform Design Based on Reproducing Kernel

H. Du, M. Shi, J. Zhang and X. Jiang

Short Supported Biorthogonal Multiwavelets System with High

Vanishing Moments Associated with Hermite Interpolant Function

L. H. Cui, Z. X. Cheng and J. S. Leng

Assessing Harmonic Impedance by Synchronous Wavelet Transform

A. Zhang and $H$. Yang

Study on Bifurcation Phenomena in a Buck-Boost Circuit Based on Wavelet Transform

L. Wang and X. Wei

The Criteria of Weak Generalized Localization for Multiple WalshFourier Series of Functions in Orlicz Classes

S. K. Bloshanskaya

The Algorithm Research of Fast Multiwavelet Transform Based on FFT M. Wang and J. He

Precursors of Engine Failures Revealed by Wavelet Analysis

I. M. Dremin

Beam Bending Analysis Using Wavelet Finite Element

B. Li, X. Chen, Z. He and J. Zhuo

Transient Thermal Analysis of Duplicating Paper Using Wavelet on the Interval Elements

$X$. Chen, B. Li, S. Yang and Z. He

A New Method to Choose the Thresholds in Wavelet Denoising

S. He, S. Yang and L. Wu

The Application of Wavelet for Passive Location Using TDOA

Information

M. Zhu, Z. You and J. Nie

A Novel Multiple Resolution Analysis Method

H. Yang, X. Miao, W. He and Z. Ren 
Application of Wavelet Neural Network for Automatic Ranging Cutting Height of Shearer

T. Yang and S. Xiong

The Properties of Biorthogonnal Multiwavelet Packets with Scale $=\mathrm{a}$

J. S. Leng, Z. Cheng and J. Li

The Application of the Wavelet Transform to the Discrete

Data of Logging

S. Zeng, B. Chen, X. Yang, J. Zhao and C. Xu

PN Code Acquisition Detection for CDMA Networks Based on

Wavelet Transform and Artificial Neural Network $X$. Zeng and $X$. Tan

Study on the Application of Wavelet Transform in Detection of Low Observable Targets

F. Su, C. Qu, Y. He and M. Xia 


\section{Contents}

(Volume 2)

\section{Keynote Presentations}

Demodulation by Complex-Valued Wavelets for Stochastic Pattern

Recognition: How Iris Recognition Works

J. Daugman

\section{Image Compression and Coding}

Study of Image Wavelet De-Noising and Compression Scheme

D. Ma, Z. Chen, H. Zhang and S. Yang

Meaningful Image Digital Watermarking Scheme Based on Multi-

Channel Watermarking Model in Wavelet Domain

J. Zhang, R. Xie, H. Hu and H. Z. Hu

Wavelets and Image Compression

V. A. Nechitailo

A New JPEG2000 Region-of-Interest Image Coding Method:

Foveal Shift

$J$. Zheng and L. Y. Fan

The Hierarchically Weighted Wavelet Image Coding Based on SPIHT J. L. Zhang, X. Z. Liang and H. Q. Sun

Stripe-Based Wavelet Transform and its Coding $X$. Zhang, L. Cheng and $K$. $L i$

Wavelet Lossy and Lossless Compression Techniques for Image

W. Pan, Q. Lin, J. Li, H. Wang and J. Zhai

\section{Video Coding and Processing}

Fast Wavelet-Based Video Codec and its Application in an IP Version 6-Ready Serverless Videoconferencing 
A New Time-Frequency Mapping Structure for High Quality Audio

Coding

J. X. Yan, Z. W. Dong and W. B. Dou

\section{Theory}

A Combined Approach to Separate Atrial Fibrillation Wave

Z. Y. Lee, J. H. Yu, T. Y. Xiang, H. X. Ma, Y. H. Niu, Z. X. Xie and J. C. Yang

Pre-Processing Design for Multiwavelet Filters Using Neural Networks

R. Ashino and A. Morimoto

Wavelet Representation of Fractal Measures in Weighted Wiener Amalgam Spaces

S. S. Pandey

Domain Decomposition Adaptive Algorithm with Wavelet Method to Trace Shock Wave

$B$. Wu and $X$. Liu

Fast Algorithm of Wavelet Transform on Real Time Signal F. Han, H. Li, X. Huang and C. Liao

Biorthogonal Vector Filter Banks via Hermite Interpolation and Lifting Scheme

P. Shui and Z. Bao

A Stepwise Updating Algorithm for Multiresolution Wavelet Neural Networks

Q. Sui and Y. Gao

On a Class of Optimal Wavelets

N. A. Strelkov and V. L. Dol'nikov

An Iris Recognition System Including Acquisition and Locating as well as Texture Representation

Y. Liu, X. Ming, S. Yuan and Z. Li

A Criterion of Orthogonal for a Class of Scaling Functions with

Dilation Factor 3

W. Ye

A New Orthogonal Multiwavelet Network for Function Approximation $J$. Wang, X. Shang and G. Song 
The Construction of Biorthogonal Multi-Scaling Functions Possessing Higher Approximation Order with Fractal Interpolation Functions

$B$. Zhang, J. Wang and G. Song

Application of Wavelet De-Noising and Multiresolution Analysis in the Recognition of Turbulent Coherent Structure

$X$. Wang, J. Teng, Y. Lian and N. Yurchenko

Multiwavelet-Based Differentiation Matrix with Absorbed Boundary

Conditions

X. C. Feng, G. Song and Y. Y. Tang

A Class of Non-Separable Bivariate Biorthogonal Scaling Functions with Compact Support

$G$. Wang and Z. X. Cheng

A New Image Enhancement and Object Obtaining Method in the Environment of Low Contrast and Strong Background Noise

J. Yan, M. Zhu, Y. Wang and $Y$. Li

Generic Programming with Reusable Wavelet Transforms

W. Yuan and J. Guo

Construction on Orthogonal Multiwavelets for Derivatives of

Multivariate Vector Scaling Function with Compact Support

$X$. Feng and $Z$. Cheng

FPGA Implementation of 1-D Discrete Wavelet Transform

Z. Xie, G. Yan, G. Lv and Z. Wei

An Application of Continuous Wavelet Transform in $L^{2}\left(R^{n}\right)$

H. $Q u$ and C. Xu

Computation of the Bivariate Wavelet Function Value with Arbitrary

Dilation Matrix

C. Xie, S. Yang and Z. Cheng

\section{Image Processing}

A Wavelet-Based Digital Watermarking Algorithm

H. Q. Sun, X. Z. Liang and J. L. Zhang

Digital Watermark Based on Wavelet Transform for Audio Signals

$Y$. Wang, S. Wang and J. Zhang 
FOPEN Ultra-Wide Band SAR Imaging Based on Wavelet Interpolation

H. Guo, X. Fang, Y. Wang and D. Liang

Biorthogonal Wavelets Research Based on Lifting Scheme

Y. Wu, Q. Pan, H. Zhang and Y. Zhang

The Application Research of Wavelet Analysis in Medical Image

Processing

G. Xie, Y. Wang and L. Ming

Segmentation of Ultrasonic Medical Image Based on Wavelet

Transform and Mathematical Morphology

Q. Liu and T. Wang

An Wavelet-Fractal Neural Network Used in Cutting Tools Wear Monitoring

P. Xie and B. Liu

Texture Segmentation Based on Wavelet Textons

P. Zhang, S. Zhao and S. Peng

The Fingerprint Image Noise Reducing and Minutia Matching in

Verification

S. Jin, M. Zeng and D. Chen

A Robust Digital Watermarking System by Specifying the Embedded Position Using Wavelet Transform

$Y$. Ueno and K. Murakami

Inter-Scale Dependency Based Adaptive Shrinkage De-Noising for Image

S. Wang, D. Zou, S. Shen and Z. Fang

Speckle Reduction in SAR Image Using Wavelet

M. Zhu, Z. You and J. Nie

Watermarking in Image Edges

799

$W$. Wang, $Y$. Y. Tang and $G$. Xu

\section{Signal Processing}

Research of the Gyro Signal De-Noising Method Based on Stationary Wavelets Transform 
On the Security for the Fail-Stop Digital Signatures

Q. Zhang, J. M. Zheng and C. Yang

Adaptive De-Noising of Low SNR Signals

D. Isar and A. Isar

A Resolution Comparison in the Wavelet Transform and Several TimeFrequency Representations

C. $Q u, Y$. He and F. Su

Improving Signal-Noise Rate of Seismic Data by Balanced Orthogonal Multiwavelet Transform

W. Z. He and A. D. Wu

Wavelet Domain Wiener Filter and its Application in Signal Denoising Q. Li, J. Teng, $Q$. Zhao and S. $L i$

Performance Analysis of Wavelet Packets Multicarrier CDMA in Rayleigh Fading Channel

M. Li, Q. Peng, S. Zhong and Y. Liu

Research on Fractal Scaleless Range of Vibration Signals Based on Genetic Algorithm

G. Ren, H. Zheng, Y. Zhang, L. Tang and J. Gao

Wavelets Analysis on the Feature Collection and the Influencing Factors of the Surface Roughness in Vibratory Machining

L. Z. Gu, L. H. Chen and F. S. Bai

Fractal Application in Analyzing Vibration Signals of Diesel Engine

L. Tang, H. Feng, W. Wang and H. Wang

\section{Systems and Application}

Electronic Signature System Based on Wavelet Analysis (Abstract) $J . L i$

A Special Poisson Sum and its Applications

Y. Cen, D. Sun, Y. Wei and L. Cen

Nonlinear Generalized Predictive Control Based on Inverse System

Using Wavelet Networks

$J$. Guo, Q. Chen, W. Hu and $S . X u$

Predicting Protein Structural Features with Wavelet Packet 
Construction of 2-D Mother Wavelet from 1-D Mother Wavelet by Inverse Projecting

L. Zeng, P. Wang and Y. Y. Tang

Wavelet Singularity Analysis of Radar Target Disappearing and

Capture

X. Huang, B. Gao, F. Han, R. Yuan, C. Liao and Z. Che

Exact Solutions for Rotation Fluid Equation

Z. Huang and L. Xia

Starting Characteristic Testing and Analysis of Ultrasonic Motor Based on Wavelet Transformation

S. L. Shen and C. S. Zhao

Extraction of the Mutative Rule of Stock Index Using Singularity

Detection Technology

W. Bao, S. Zhou, Y. Luo, D. Feng and X. Guo

Predictive Models of Seasonal Time Series for Hemorrhagicfever with Renal Syndrome Based on Wavelet Analysis

X. Guo, J. Lin, W. Cao, Y. Luo, S. Zhou, W. Bao and Y. Cheng

Fault Diagnosis Based on Double Wavelet Neural Network

G. Li, X. Qi and L. Yao

VEP Signal Extraction Using Wavelet in Brain-Computer Interface Research

Q. He, C. Peng, B. Wu, H. Wang and L. Zhu

Fault Feature Extraction of Hydro-Generator Vibration Signals Based on Wavelet Shrinkage

T. Sun, T. S. Huang, M. Li, F. X. Sun and J. D. Xiang

Wavelet Method Based on MPARIMA Model for STLF

Q.W. Ran, Q. Wang, Y. Z. Shan, D. S. Yueng and C. C. Tseng

Application Study for Identifying Nuclear Explosion from Earthquake with Wavelet Packet Method

$X$. Yang, $P$. Shen and Z. Zheng

An Application of Wavelet Analysis to the Spillovers of Stock Markets in China

$$
\text { C. Su, X. Liu and L. Liu }
$$

Investigating the Non-Linear Behaviors of Rub-Impact Rotor System with Wavelet Scalogram

Z. K. Peng, Y. Y. He, Q. Lu, W. X. Lu and F. L. Chu 
A Software System for Analog-Circuit Fault Diagnosis Based on Wavelet Analysis

X. Luo, J. Cai and C. Shang

Analysis of the DLA-Process with Gravitational Interaction of Particles and Growing Cluster

A. Loskutov, D. Andrievsky, V. Ivanov and A. Ryabov

Vehicle Detection on Highway Based on Direction-Fractal Dimension

$X$. Zhou, X. Huang, C. Liao and Y. Qiao

Digital Watermark

Q. Li, Q. Meng, W. Gao and S. Yang

Fractal Application for Predicting Protein Subcellular Location

K. He, Z. D. Huang, L. He and G. B. Zhou 